2 Adshead S, du Feu M. Mental health service provision for the deaf community. Progress Neurol Psychiatry 2005; 9: 26-30.

3 Munoz-Baell IM, Ruiz MT. Empowering the deaf. Let the deaf be deaf. $J$ Epidemiol Comm Health 2000; 54: 40-4.

4 Ralston E, Zazove P, Gorenflo DW. Physicians' attitudes and beliefs about deaf patients. J Am Board Fam Practice 1996; 9: 167-73.

5 Department of Health. Mental Health and Deafness - Towards Equity and Access: Best Practice Guidance. Department of Health, 2005.

Sara L. Adshead, Consultant Psychiatrist (formerly locum consultant psychiatrist), National Deaf Mental Health Service, Leamington Spa, Warwickshire, UK, email: sara.adshead@covwarkpt.nhs.uk

doi: $10.1192 / \mathrm{pb} .37 .1 .36 \mathrm{a}$

\section{The impact of the Health and Social Care Act 2012 on forensic psychiatry}

As a National Health Service forensic psychiatrist working on a newly commissioned low secure ward, the statement: 'it is all too predictable that yet more patients will be pushed down forensic care pathways from which return to mainstream care will be difficult (p. 402)' in Holloway's excellent November editorial $^{1}$ struck a firm chord with me.

In the past year, I have overseen an expansion of both the low secure forensic estate and the out of area patient placements. Although there was some clinical and commissioning intent to introduce the low secure estate to allow transition out of the medium secure estate (and indeed this has happened to some extent), there has been quite a surge of patients coming from the general acute services and the community.

We also receive some prison transfers; these include general adult community patients with no prior forensic history who were missed in the community owing to (poorly resourced) service lapses. Such patients become 'forensic' because of a lack of adequate community psychiatric services rather than being appropriate referrals to the service. In any case, we are expanding.

Good news for forensic staff, but not so good for patient care. Earlier psychiatric intervention for them may have even saved them from being locked up in prison. This is low-income country psychiatry in a high-income country.

At a recent presentation by some Californian psychiatrists, I was very impressed by the vigour with which they grapple with often very difficult legal circumstances of psychiatric care in their jurisdiction. They noted that most of their state hospital beds were occupied by their forensic patients. There was very little available for non-forensic patients, either in hospital or in the community. I wonder whether here in England we are also heading in that direction.

Finally, it appears that in this evolving, risk-focused, forensic-heavy psychiatric care environment, the 'forensic' patient today is not the same forensic patient from 20 years ago. These days, not every forensic patient is a high secure step-down patient. Why is it then more difficult to discharge forensic patients into the community, and return them to mainstream services? At the very least, the expanding low secure estate ought to provide an easier interface within the psychiatric services than was the case in the past. This way we will have done our best for our patients while contending with the difficult care environment being planned for us by this government. Indeed, who else will?

1 Holloway F. The Health and Social Care Act 2012: what will it mean for mental health services in England? Psychiatrist 2012; 36: 401-3.

Muzaffar Husain, Consultant Forensic Psychiatrist, Kent Forensic Psychiatric Service, Beckenham, Kent, UK, email is Muzaffar.Husain@ kmpt.nhs.uk

doi: $10.1192 / p b .37 .1 .37$

\section{Needless complexity in commissioning}

Having attended a local third-sector and service user conference and having read the editorial by Holloway, ${ }^{1}$ I wonder whether the following needs more consideration.

It strikes me that dividing mental health commissioning responsibilities locally between the clinical commissioning groups (mental illness treatments) and local authorities (suicide and substance misuse prevention, mental health promotion) poses unnecessary complexity and bureaucratic waste. Despite lay representation in clinical commissioning groups, there is no democratic accountability similar to that offered through local councillors and local authority scrutiny committees which can call providers to attend a public meeting to account for their priorities in using public funds. Perhaps local elections might be more popular if electors realise that councillors could be voted out if they are not active in championing mental health issues such as dementia care. Furthermore, local authorities already have experienced procurement teams with ready access to performance management and audit functions.

Therefore, I wonder whether clinical commissioning groups should be relieved of all mental health commissioning responsibilities, with this function carried out entirely by local authorities. This would allow the commissioning groups to concentrate on acute and chronic medical diseases (which contribute to most of the cost via hospital bed usage and new technology). The added benefit of mental health being commissioned by local authorities would be integration of social and healthcare budgets for the benefit of people with severe mental illness such as psychosis and dementia. As a practising clinician, I find it difficult to separate social and health interventions in providing a good outcome for an individual patient; usually, there is a synergistic effect.

The other issue discussed by Holloway is 'personalisation'. It is hoped that by April 2013, 70\% of eligible mental health service users (mainly with severe chronic illness) will have a personal budget with an allocated broker to help clarify and achieve their choices in interventions. The above rationalising of commissioning would lend itself to a combined health and social care budget which can be spent pragmatically. A chipand-pin charge card could be introduced to carry a combined budget, with greater accountability and freedom from having to collect receipts.

The third issue highlighted at the conference was an increasing body of evidence suggesting that active collaborations between statutory mental health providers and third-sector organisations result in better outcomes and lower number of bed days in psychiatric hospitals. Perhaps this should be considered an essential requirement for mental health trusts when submitting bids for a service. 